\title{
Convergence of a Semi-Discrete \\ Kinetic Scheme for the System of Isentropic Gas Dynamics with $\gamma=3$
}

\section{Alexis Vasseur}

ABSTRACT. We consider, for the system of isentropic gas dynamics with $\gamma=3$, a time-discrete kinetic scheme, closely related to the kinetic formulation of Lions, Perthame, and Tadmor. We prove the convergence of this scheme by using averaging lemmas and we show that the convergence is strong in $L_{\text {loc }}^{1}$, even at the microscopic level.

1. Introduction. We consider the equations of isentropic gas dynamics with $\gamma=3$. In Eulerian coordinates, these equations form a $2 \times 2$ hyperbolic system of nonlinear conservation laws

$$
\left\{\begin{array}{l}
\partial_{t} \rho+\partial_{x} \rho u=0 \\
\partial_{t}(\rho u)+\partial_{x}\left(\rho u^{2}+\frac{\rho^{3}}{12}\right)=0, \quad t \geq 0, x \in \mathbb{R}
\end{array}\right.
$$

where the unknowns $\rho(t, x) \geq 0$ and $\rho u(t, x)$ are respectively the density and the momentum of the gas. They are given at time $t=0$ by the initial data $\rho^{0}(x)$ and $\rho^{0} u^{0}(x)$. As usual, we are concerned only with entropy-admissible solutions of (1) which verify

$$
\partial_{t} \eta(\rho, \rho u)+\partial_{x} H(\rho, \rho u) \leq 0
$$

in the sense of distributions for every pair $(\eta, H)$ of entropy and corresponding entropy flux.

The general idea of kinetic schemes (often called Boltzmann schemes, see [12] for example) is old and closely related to the classical and formal derivation of the Euler equations from the Boltzmann equation. In this kinetic derivation, the macroscopic conservation laws and related entropy conditions of the Euler equations are directly recovered from the momenta with respect to the velocity 
variable $v$ of the Boltzmann equation in the limit when the collision rate goes to infinity, which forces the microscopic density $f(t, x, v) \geq 0$ to be in a so-called Maxwellian equilibrium state, namely a Gaussian function in $v$. In the same way, a time-discrete kinetic scheme for (1), with time step $\Delta t>0$, is supposed to provide an approximate solution of (1), obtained as the two first momenta of a microscopic density $f_{\Delta t}(t, x, v) \geq 0$, depending on an additional variable $v \in \mathbb{R}$, and solution to a suitable kinetic equation. To achieve that, we need a suitable concept of equilibrium, or Maxwellian function, associated, for each fixed $(t, x)$, with $f(t, x, \cdot)$. Instead of Gaussian functions, it is enough to use simpler functions, such as step functions (following Kaniel and Falcovitz [13], in the special case $\gamma=3$ ):

Definition 1.1. Given a microscopic function $f(v) \geq 0$, bounded and supported in some interval $[0, L]$, we define the associated Maxwellian or equilibrium density

$$
\mathcal{M} f(v)=\mathbf{1}_{|v-u| \leq \rho / 2}
$$

where

$$
\left\{\begin{aligned}
\rho & =\int_{0}^{L} f(v) d v \\
\rho u & =\int_{0}^{L} v f(v) d v
\end{aligned}\right.
$$

We define our kinetic approximation as a microscopic density $f_{\Delta t}(t, x, v) \geq 0$, subject to the following rule: on each interval $t \in] n \Delta t ;(n+1) \Delta t[$, the free transport equation

$$
\partial_{t} f_{\Delta t}+v \partial_{x} f_{\Delta t}=0
$$

is satisfied and, at every time step $t=n \Delta t$ and each point $x$, the associated Maxwellian function is substituted for $f_{\Delta t}(t-0, x, \cdot)$. More precisely, denoting $t_{\Delta t}=E(t / \Delta t) \Delta t$, where $E$ stands for the integer part, we set:

$$
\left\{\begin{aligned}
f_{\Delta t}(t, x, v) & =f_{\Delta t}\left(t_{\Delta t}, x-\left(t-t_{\Delta t}\right) v, v\right), \\
f_{\Delta t}\left(t_{\Delta t}+\Delta t, x, v\right) & =\mathcal{M}\left(f_{\Delta t}\left(t_{\Delta t}, x-\Delta t v, v\right)\right) .
\end{aligned}\right.
$$

As recently observed [2], this semi-discrete scheme, which is somewhat reminiscent of Chorin's projection method in incompressible fluid mechanics [7], corresponds to the transport-collapse method for systems of conservation laws defined in [4] and [14]. It can also be seen as the time-discrete version of the Lions, Perthame, and Tadmor [16] kinetic formulation of system (1). Let us recall that this formulation involves a microscopic density function $f(t, x, v)$ subject to solve 


$$
\partial_{t} f+v \partial_{x} f=-\partial_{v}^{2} \mu \quad \text { in } \mathbb{R}^{+} \times \mathbb{R} \times[0, L]
$$

with the constraint:

$$
f(t, x, v)=\mathcal{M} f(t, x, v) \text { a.e. }
$$

where $\mu(t, x, v)$ is a nonnegative bounded measure, and $\mathcal{M} f$ is the Maxwellian function related to $f$ with respect to the variable $v$ defined in Definition 1.1. When we multiply equation $(5)$ by the vector $(1, v)$ and integrate it with respect to $v$, we exactly recover (1) for the momenta

$$
\left\{\begin{aligned}
\rho(t, x) & =\int_{0}^{L} f(t, x, v) d v \\
\rho(t, x) u(t, x) & =\int_{0}^{L} v f(t, x, v) d v
\end{aligned}\right.
$$

since, according to Definition 1.1,

$$
\mathcal{M} f(t, x, v)=\mathbf{1}_{|v-u(t, x)| \leq \rho(t, x) / 2} .
$$

Moreover, when we multiply equation (5) by a convex function $\varphi(v)$ and we integrate with respect to $v$, we recover the entropy inequalities (2). As a matter of fact, it is shown in [16] that $f$ is solution of (5) if and only if $(\rho, \rho u)=\left(\int f d v, \int v f d v\right)$ is solution of $(1)(2)$. In this framework (in the special case $\gamma=3$ ), the existence of global solutions is an easy consequence of the strong $L_{\text {loc }}^{p}$ compactness of the momenta, which follows from a sharpened version [9] of the so-called averaging lemma, a powerful tool in kinetic theory, independently introduced by Agoshkov [1] and Golse, Perthame, and Sentis [11]. Let us emphasize that the case $\gamma=3$ is very peculiar in the framework of conservation laws [17]. Indeed, when there is no shock, the two Riemann invariants $b=\rho / 2+(\rho u) / \rho$ and $a=\rho / 2-(\rho u) / \rho$ are solutions of the inviscid Burgers equation. However, when shocks appear, evolution equations of $a$ and $b$ are not un-coupled anymore. In fact, they do not verify the Rankine-Hugoniot conditions associated to the inviscid Burgers equation. Moreover, despite its simplicity, this system does not belong to the Temple class and bounded variation analysis cannot be used for general initial data. Thus, it is natural to use purely kinetic tools, such as averaging lemma, to prove the convergence of the time-discrete kinetic scheme. Quite surprisingly, this is not an easy extension of [16], essentially because the approximate density function $f_{\Delta}$ is a Maxwellian function only at discrete times $t=n \Delta t$ and can a priori be very far from equilibrium at other times. The aim of this paper is precisely to investigate and solve this difficulty. (Notice that relatedbut different-problems have been observed and investigated by L. Desvillettes and S. Mischler for a time-discrete approximation of the Boltzmann equations [8].) More precisely, we will show the following theorem. 
Theorem 1.1. There exists a sequence $\Delta t_{n}$, which converges to 0 , such that $f_{\Delta t_{n}}$ converges strongly in $L_{\mathrm{loc}}^{1}\left(\mathbb{R}^{+} \times \mathbb{R} \times[0, L]\right)$ to $f$ and $f=\mathcal{M} f$. Therefore $f$ is a solution of kinetic equation (5). Moreover, if $f^{0}$ is Maxwellian with respect to the $v$ variable,

$$
\lim _{t \mapsto 0}\left\|f(t, \cdot, \cdot)-f^{0}(\cdot, \cdot)\right\|_{L^{1}(\mathbb{R} \times[0, L])}=0 .
$$

Since the convergence holds in $L_{\text {loc }}^{1}\left(\mathbb{R}^{+} \times \mathbb{R} \times[0, L]\right)$, we could expect difficulties to recover in a good sense the initial value for $t=0$. The last statement of the theorem ensures that $f$ is continuous at time $t=0$ in $L^{1}(\mathbb{R} \times[0, L])$ and that $f(t=0)=f^{0}$.

Let us now sketch the method of the proof of Theorem 1.1. The difficulty lies in showing that $f=\mathcal{M} f$ at the limit. Indeed, as mentioned earlier, $f_{\Delta t}$ is Maxwellian at every time $n \Delta t$ for $n \in \mathbb{N}$, but it has no reason to be still Maxwellian between such times. Therefore, in order to show that the limit is Maxwellian almost everywhere, we have to study very carefully the behavior of $f_{\Delta t}$ on those intervals. To do so, we introduce the following rescaled microscopic function of $f_{\Delta t}$ about a fixed $(t, x)$.

$$
\bar{f}_{\Delta t_{n}}(t, x, v, \tau, \xi)=f_{\Delta t_{n}}\left(t_{\Delta t_{n}}+\tau \Delta t_{n}, x+\xi \Delta t_{n}, v\right)
$$

where $t_{\Delta t_{n}}=E\left(t / \Delta t_{n}\right) \Delta t_{n}$. We will see that the proof consists in proving that for almost every $(t, x), \bar{f}_{\Delta t_{n}}$ converges strongly to a function which does not depend anymore of $(\tau, \xi)$. This means that oscillations of length $\Delta t_{n}$ are vanishing as $\Delta t_{n}$ goes to zero.

2. General framework. It is now convenient to consider the kinetic model of Brenier and Corrias [5], which includes both the inviscid Burgers equation and the system of isentropic gas dynamics with $\gamma=3$. This will provide us a good framework to prove Theorem 1.1. (Originally, the aim of Brenier and Corrias was to define a rigorous concept of entropy multi-valued solutions with at most $K$ branches for the inviscid Burgers equation.) It is described in the following way. First we fix a positive integer $K$. Then we consider a general notion of Maxwellian function which includes the previous one for $K=2$.

Definition 2.1. Let $f \in L^{\infty}([0, L])$ such that for almost every $v \in[0, L]$

$$
0 \leq f(v) \leq 1
$$

We say that $\mathcal{M} f$ is the Maxwellian function associated to $f$ if

$$
\begin{cases}\int_{0}^{\infty} v^{k} \mathcal{M} f(v) d v=\int_{0}^{\infty} v^{k} f(v) d v & \text { for any integer } k, 0 \leq k \leq K-1 \\ \mathcal{M} f(v) \in\{0 ; 1\} & \text { for a. e. } v, \\ T V(\mathcal{M} f(\cdot)) \leq K, & \end{cases}
$$

where $T V$ denotes the total variation on the interval $] 0, L[$. 
In [5] it is shown that the Maxwellian functions are obtained as a solution of an optimization problem.

Proposition 2.1. For every $C^{\infty}$ function $\theta$ on $[0, L]$ with

$$
\frac{\partial^{K} \theta}{\partial v^{K}}(v) \geq 0
$$

the Maxwellian function $v \rightarrow \mathcal{M} f(v)$ is the unique minimizer of $\int_{0}^{\infty} \theta(v) g(v) d v$ among all functions $g$ valued in $[0,1]$ such that $\int_{0}^{\infty} v^{k} g(v) d v=\int_{0}^{\infty} v^{k} f(v) d v$ for $k=0, \ldots, K-1$. Especially we can take $\theta(v)=v^{K}$.

The kinetic model is then described as follows. We are looking for $f \in L^{\infty}\left(\mathbb{R}^{+} \times\right.$ $\mathbb{R} \times[0, L])$, solution of

$$
\partial_{t} f+v \partial_{x} f=(-1)^{K-1} \partial_{v}^{K} \mu \text { in } \mathbb{R}^{+} \times \mathbb{R} \times[0, L],
$$

with the constraint:

$$
f(t, x, v)=\mathcal{M} f(t, x, v) \text { a.e. }
$$

where $\mu(t, x, v)$ is a nonnegative bounded measure, and $\mathcal{M} f$ is the Maxwellian function related to $f$ with respect to the variable $v$ defined in Definition 2.1.

Then we consider the kinetic semi-discrete scheme defined in the introduction by (4), where $\mathcal{M} f$ is the Maxwellian function defined in (6). When $K=2$, Definition 1.1 is exactly the same that Definition 2.1. So equation (7) is the kinetic formulation (5) of the isentropic gas dynamic system with $\gamma=3$ described in the introduction. The case $K=1$ corresponds to the inviscid Burgers equation. In fact, let us write $u_{\Delta t}(t, x)=\int_{0}^{\infty} f_{\Delta t}(t, x, v) d v$. Then, according to (6),

$$
\mathcal{M} f_{\Delta t}(t, x, v)=\mathbf{1}_{\left[0, u_{\Delta t}(t, x)\right]}(v) .
$$

So if we integrate equation (7) in respect of $v$ we find

$$
\partial_{t} u+\partial_{x} \frac{u^{2}}{2}=0
$$

Moreover, for every convex function $\varphi$, if we multiply the equation (7) by $\varphi^{\prime}(v)$ and we integrate it with respect to $v$, we find

$$
\partial_{t} \varphi(u)+\partial_{x} \psi(u) \leq 0
$$

with $\psi^{\prime}(v)=v \varphi^{\prime}(v)$. Therefore $u$ is the Kruzkov solution of inviscid Burgers equation. Indeed, in this case, the Maxwellian structure (8) is the one introduced in $[3,4]$ for scalar conservation laws and equation (7) is nothing but the kinetic formulation of the inviscid Burgers equation introduced by P. L. Lions, 
B. Perthame, and E. Tadmor in [15]. We can write the kinetic scheme (4) in the following way.

For all $t \in[n \Delta t,(n+1) \Delta t[$

$$
u_{\Delta t}(t, x)=\int_{0}^{\infty} \mathbf{1}_{\left\{0 \leq v \leq u_{\Delta t}(n \Delta t, x-(t-n \Delta t) v)\right\}}(v) d v .
$$

Then we easily see that this scheme is nothing but the "transport-collapse" method [3, 4] for the inviscid Burgers equation (see also [10]). It is known in this case that

$$
\text { for all } n, \quad T V_{x}\left(u_{\Delta t}((n+1) \Delta t, \cdot)\right) \leq T V_{x}\left(u_{\Delta t}(n \Delta t, \cdot)\right)
$$

(where $T V$ denotes the total variation). Then the convergence of $u_{\Delta t}$ to $u$ can be proved by using classical compactness tools. However, in [18] we have given numerical experiments which show that the scheme is no longer total variation diminishing for $K \geq 2$, so this proof can not be extended to the general case. We have provided in [18] an alternative proof for the case $K=1$, using averaging lemmas. Unfortunately, this proof is not valid for $K \geq 2$ anymore.

From now on we will consider Definition 2.1 of Maxwellian function and kinetic model (7). The following is devoted to the proof of Theorem 1.1 in this framework, namely the proof of the convergence of scheme (4).

3. Strong convergence of Maxwellian functions. First we will show some links between Maxwellian functions and their $K-1$ first momenta. Finally we will end this section showing some properties of $f_{\Delta t}$ and $\mathcal{M} f_{\Delta t}$.

Proposition 3.1. Let $\mathcal{A}$ be an open set of an Euclidean space equipped with the Lebesgue measure, $g_{n} \in L^{\infty}(\mathcal{A} \times[0, L])$ with $0 \leq g_{n}(a, v) \leq 1$, and $g \in L^{\infty}(\mathcal{A} \times[0, L])$ with $0 \leq g(a, v) \leq 1$ such that $\int_{0}^{L} v^{k} g_{n}(\cdot, v) d v$ converges for all integers $0 \leq k<K$ to $\int_{0}^{L} v^{k} g(\cdot, v) d v$ strongly in $L_{\text {loc }}^{1}(\mathcal{A})$. Then the entire family $\mathcal{M g}$ converges in $L_{\text {loc }}^{1}(\mathcal{A} \times[0, L])$ strongly to $\mathcal{M g}$.

The following straightforward corollary will be useful in the following sections.

Corollary 3.1. Let $g_{n} \in L^{\infty}(\mathcal{A} \times[0, L])$ with $0 \leq g_{n}(a, v) \leq 1$ and $g \in$ $L^{\infty}(\mathcal{A} \times[0, L])$ such that $g_{n}$ converges strongly in $L_{\text {loc }}^{1}(\mathcal{A} \times[0, L])$ to $g$. Then $\mathcal{M g}$ converges in $L_{\text {loc }}^{1}(\mathcal{A} \times[0, L])$ strongly to $\mathcal{M g}$.

Proof of Proposition 3.1. First, there exists a subsequence $n_{p}$ such that for almost every $a \in \mathcal{A}$ we have $\int_{0}^{L} v^{k} \mathcal{M} g_{n}(a, v) d v$ which converges for all integers $0 \leq k<K$ to $\int_{0}^{L} v^{k} g(a, v) d v$. But according to (6), we have

$$
T V_{v}\left(\mathcal{M} g_{n_{p}}(a, \cdot)\right) \leq K .
$$


Therefore there exists a subsequence depending on $a$, denoted $n_{p}(a)$, such that $\mathcal{M} g_{n_{p}(a)}(a, \cdot)$ converges in $L^{1}([0, L])$ strongly to a function $h(a, \cdot)$. So up to a further extraction (still depending on $a), \mathcal{M} g_{n_{p}(a)}(a, \cdot)$ converges for almost every $v \in[0, L]$, which shows that $h(a, \cdot)$ is valued in $\{0,1\}$ and that $T V_{v}(h(a, \cdot)) \leq K$. Hence $h$ is Maxwellian with respect to $v$. Moreover, for all integers $0 \leq k<K$, we have

$$
\int_{0}^{L} v^{k} \mathcal{M} g_{n}(a, v) d v=\int_{0}^{L} v^{k} g_{n}(a, v) d v
$$

and therefore

$$
\int_{0}^{L} v^{k} h(a, v) d v=\int_{0}^{L} v^{k} g(a, v) d v
$$

as $n \rightarrow+\infty$. So $h=\mathcal{M g}$ according to (6). Finally, by the uniqueness of the limit, the entire sequence $\mathcal{M} g_{n_{p}}(a, \cdot)$ converges strongly in $L^{1}([0, L])$ to $\mathcal{M} g(a, \cdot)$. Since those functions are uniformly bounded in $L^{\infty}(\mathcal{A} \times[0, L])$, using Lebesgue's dominated convergence theorem, we find that $\mathcal{M} g_{n_{p}}$ converges strongly in $L_{\text {loc }}^{1}(\mathcal{A} \times[0, L])$ to $\mathcal{M g}$. Again by uniqueness of the limit, we find that the entire sequence $\mathcal{M} g_{n}$ converges strongly to $\mathcal{M g}$.

Let $f^{0} \in L^{1}(\mathbb{R} \times[0, L])$ be Maxwellian. We consider $f_{\Delta t}$ solution of the scheme defined by (4) and the initial data:

$$
f_{\Delta t}(0, x, v)=f^{0}(x, v)
$$

for almost every $(x, v) \in \mathbb{R} \times[0, L]$. The family $f_{\Delta t}$ has the following properties.

Proposition 3.2. The functions $f_{\Delta t}$ and $\mathcal{M} f_{\Delta t}$ lie in

$$
L^{\infty}\left(\mathbb{R}^{+} ; L^{1}(\mathbb{R} \times[0, L])\right)
$$

and for almost every $(t, x, v) \in \mathbb{R}^{+} \times \mathbb{R} \times[0, L]$ we have $0 \leq f_{\Delta t}(t, x, v) \leq 1$. Moreover, there exists a nonnegative measure $\mu_{\Delta t}(t, x, v)$ on $\mathbb{R}^{+} \times \mathbb{R} \times[0, L]$ such that

$$
\partial_{t} f_{\Delta t}+v \partial_{x} f_{\Delta t}=(-1)^{K-1} \partial_{v}^{K} \mu_{\Delta t}
$$

and we have the following estimate

$$
K ! \int_{0}^{\infty} \int_{\mathbb{R}} \int_{0}^{L} d \mu_{\Delta t}(t, x, v) \leq L^{K} \int_{\mathbb{R}} \int_{0}^{L} f^{0}(x, v) d v d x .
$$

There exists a subsequence $\Delta t_{n}$, a bounded nonnegative measure $\mu(t, x, v)$, and a function $f \in L^{\infty}\left(\mathbb{R}^{+} ; L^{1}(\mathbb{R} \times[0, L])\right)$ such that $\Delta t_{n}$ converges to 0 when $n$ tends to infinity for almost every $(t, x, v) \in \mathbb{R}^{+} \times \mathbb{R} \times[0, L], 0 \leq f(t, x, v) \leq 1$, and

$$
\begin{array}{lll}
f_{\Delta t_{n}} & \stackrel{L^{\infty} \text { weak* }}{\longrightarrow} & f \\
\mu_{\Delta t_{n}} & \stackrel{\text { measure }}{\longrightarrow} & \mu
\end{array}
$$


with:

$$
\partial_{t} f+v \partial_{x} f=(-1)^{K-1} \partial_{v}^{K} \mu .
$$

Moreover, $\mathcal{M} f_{\Delta t_{n}}$ converges strongly to $\mathcal{M f}$ in $L_{\text {loc }}^{1}\left(\mathbb{R}^{+} \times \mathbb{R} \times[0, L]\right)$.

The proof of this proposition is quite simple and is related to results in [15], [5]. It can be found in details in [18]. However, for the sake of completeness, we give a proof of this result in the appendix.

4. Local functions. In order to prove that $\mathcal{M} f(t, x, v)=f(t, x, v)$, we have to study the behavior of $f_{\Delta t}(t, x, v)$ on the periods of time

$$
t \in] n \Delta t,(n+1) \Delta t[.
$$

To do so, we introduce a new function which describes the behavior of $f_{\Delta t}$ about a fixed $(t, x)$.

Definition 4.1. For every sequence of functions $g_{\Delta t_{n}} \in L^{\infty}\left(\mathbb{R}^{+} \times \mathbb{R} \times[0, L]\right)$ we associate a sequence of local functions $\bar{g}_{\Delta t_{n}}$, which depend of

$$
(t, x, v, \tau, \xi) \in \mathbb{R}^{+} \times \mathbb{R} \times[0, L] \times[0,1] \times \mathbb{R}
$$

and which are defined by

$$
\bar{g}_{\Delta t_{n}}(t, x, v, \tau, \xi)=g_{\Delta t_{n}}\left(t_{\Delta t_{n}}+\tau \Delta t_{n}, x+\xi \Delta t_{n}, v\right),
$$

where $t_{\Delta t_{n}}=E\left(t / \Delta t_{n}\right) \Delta t_{n}$.

We introduce tools to compare the convergence of $\bar{f}_{\Delta t_{n}}$ and the convergence of $f_{\Delta t_{n}}$ when $\Delta t_{n}$ converges to 0 . Let us first show the following elementary lemma.

Lemma 4.1. Let $\varphi \in L^{1}([0, T])$ and

$$
\varepsilon_{n}(\varphi)=\int_{0}^{T} \int_{0}^{1}\left|\varphi\left(t_{\Delta t_{n}}+\tau \Delta t_{n}\right)-\varphi(t)\right| d \tau d t .
$$

Then $\varepsilon_{n}(\varphi)$ converges to zero as $n$ tends to infinity.

Proof. The proof is obvious when $\varphi \in C^{0}([0, T])$. We conclude by observing that if we approximate $\varphi \in L^{1}([0, T])$ by $\tilde{\varphi} \in C^{0}([0, T])$,

$$
\begin{aligned}
\left|\varepsilon_{n}(\varphi)-\varepsilon_{n}(\tilde{\varphi})\right| & \leq \int_{0}^{T} \int_{0}^{1}|\varphi-\tilde{\varphi}|\left(t_{\Delta t_{n}}+\tau \Delta t_{n}\right) d \tau d t+\int_{0}^{T}|\varphi-\tilde{\varphi}|(t) d t \\
& =\sum_{m \Delta t_{n} \leq T} \Delta t_{n} \int_{0}^{1}|\varphi-\tilde{\varphi}|\left(m \Delta t_{n}+\tau \Delta t_{n}\right) d \tau+\int_{0}^{T}|\varphi-\tilde{\varphi}|(t) d t \\
& \leq 2\|\varphi-\tilde{\varphi}\|_{L^{1}([0, T])^{\circ}}
\end{aligned}
$$

We are now able to show the following proposition which is the main point of this section. 
Proposition 4.1. Let $g_{\Delta t_{n}} \in L^{\infty}\left(\mathbb{R}^{+} \times \mathbb{R} \times[0, L]\right)$ be a sequence of functions with $0 \leq g_{\Delta t_{n}}(t, x, v) \leq 1$ and $\bar{g}_{\Delta t_{n}}$ the associated sequence of local functions. Let $g$ be a function in $L^{\infty}\left(\mathbb{R}^{+} \times \mathbb{R} \times[0, L]\right)$ with $0 \leq g(t, x, v) \leq 1$. Then

$$
\int_{0}^{T} \int_{-R}^{R} \int_{0}^{L}\left|g_{\Delta t_{n}}(t, x, v)-g(t, x, v)\right| d v d x d t \stackrel{n \mapsto+\infty}{\longrightarrow} 0
$$

for every $R>0$ and $T>0$ if and only if

$$
\int_{0}^{T} \int_{-R}^{R} \int_{0}^{L} \int_{0}^{1} \int_{-X}^{X}\left|\bar{g}_{\Delta t_{n}}(t, x, v, \tau, \xi)-g(t, x, v)\right| d \xi d \tau d v d x d t \stackrel{n \mapsto+\infty}{\longrightarrow} 0
$$

for every $R>0, X>0$, and $T>0$.

Proof. Let us denote $\mathcal{O}=[0, T] \times[-R, R] \times[0, L] \times[0,1] \times[-X, X]$, where $X>0$ is fixed and

$$
\begin{aligned}
I_{n}(T)=\mid & \int_{\mathcal{O}}\left|g_{\Delta t_{n}}(t, x, v)-g(t, x, v)\right| d \xi d \tau d v d x d t \\
& \quad-\int_{\mathcal{O}}\left|\bar{g}_{\Delta t_{n}}(t, x, v, \tau, \xi)-g(t, x, v)\right| d \xi d \tau d v d x d t \mid .
\end{aligned}
$$

Then

$$
I_{n}(T) \leq I_{n}^{1}+I_{n}^{2}
$$

with

$$
\begin{aligned}
I_{n}^{1}= & \left|\int_{\mathcal{O}}\right| g_{\Delta t_{n}}-g \mid(t, x, v) d \xi d \tau d v d x d t \\
& \quad-\int_{\mathcal{O}}\left|g_{\Delta t_{n}}-g\right|\left(t_{\Delta t_{n}}+\Delta t_{n} \tau, x+\Delta t_{n} \xi, v\right) d \xi d \tau d v d x d t \mid \\
I_{n}^{2}= & \int_{\mathcal{O}}\left|g\left(t_{\Delta t_{n}}+\Delta t_{n} \tau, x+\Delta t_{n} \xi, v\right)-g(t, x, v)\right| d \xi d \tau d v d x d t .
\end{aligned}
$$

Using the change of variable $y=x+\Delta t_{n} \xi$, we find

$$
\begin{aligned}
I_{n}^{1} & \leq 8 L X^{2} T \Delta t_{n}+\left|\int_{\mathcal{O}}\right| g_{\Delta t_{n}}-g \mid(t, x, v) d \xi d \tau d v d x d t \\
& \quad-\int_{\mathcal{O}}\left|g_{\Delta t_{n}}-g\right|\left(t_{\Delta t_{n}}+\Delta t_{n} \tau, y, v\right) d \xi d \tau d v d y d t \mid \\
& \leq 8 L X(X T+R) \Delta t_{n} .
\end{aligned}
$$


Because, if we denote $T_{n}=E\left(\frac{T}{\Delta t_{n}}\right) \Delta t_{n},\left|T_{n}-T\right| \leq \Delta t_{n}$, and

$$
\begin{aligned}
\int_{0}^{T_{n}} & \int_{-R}^{R} \int_{0}^{L} \int_{-X}^{X} \int_{0}^{1}\left|g_{\Delta t_{n}}-g\right|\left(t_{\Delta t_{n}}+\Delta t_{n} \tau, y, v\right) d \xi d \tau d v d y d t \\
& =\int_{-R}^{R} \int_{0}^{L} \int_{-X}^{X} \sum_{m \Delta t_{n} \leq T} \Delta t_{n} \int_{0}^{1}\left|g_{\Delta t_{n}}-g\right|\left(m \Delta t_{n}+\tau \Delta t_{n}, x, v\right) d \tau d \xi d v d x \\
& =\int_{0}^{T_{n}} \int_{-R}^{R} \int_{0}^{L} \int_{-X}^{X}\left|g_{\Delta t_{n}}-g\right|(t, x, v) d \xi d \tau d v d x d t
\end{aligned}
$$

Finally, using again the change of variable $y=x+\Delta t_{n} \xi$ we find

$$
\begin{aligned}
I_{n}^{2} \leq & 8 L X^{2} T \Delta t_{n} \\
& +\int_{\mathcal{O}}\left|g\left(t_{\Delta t_{n}}+\Delta t_{n} \tau, y, v\right)-g(t, y, v)\right| d \xi d \tau d v d y d t \\
& +\int_{\mathcal{O}}\left|g(t, y, v)-g\left(t, y-\Delta t_{n} \xi, v\right)\right| d \xi d \tau d v d y d t .
\end{aligned}
$$

But using Lemma 4.1, for almost every $(x, v, \xi)$

$$
\lim _{n \rightarrow+\infty} \int_{0}^{T} \int_{0}^{1}\left|g\left(t_{\Delta t_{n}}+\Delta t_{n} \tau, x, v\right)-g(t, x, v)\right| d \xi d \tau d v d x d t=0 .
$$

So using Lebesgue's dominated convergence theorem, (15) converges to zero when $n$ tends to infinity. Since $g \in L^{\infty},(16)$ converges to zero too. Therefore $I_{n}^{1}$ and $I_{n}^{2}$ converge to zero. So

$$
\lim _{n \rightarrow \infty} I_{n}(T)=0
$$

which ends the proof.

5. Strong convergence of the scheme. From now on we consider the sequence $\Delta t_{n}$ defined by Proposition 3.2. We will show in this section the convergence of the scheme. Let us introduce the sequence of local functions $\bar{f}_{\Delta t_{n}}$ associated to the sequence $f_{\Delta t}$. We deduce from the properties of the functions $f_{\Delta t}$, the following ones for the corresponding local functions.

Proposition 5.1. Let $\bar{f}_{\Delta t_{n}}$ be the local function associated to $f_{\Delta t_{n}}$; there exists a set $\Omega_{1} \subset \mathbb{R}^{+} \times \mathbb{R}$ such that $\mathcal{L}\left(\mathbb{R}^{+} \times \mathbb{R} \backslash \Omega_{1}\right)=0$, where $\mathcal{L}$ denotes the Lebesgue's measure, and for every $(t, x) \in \Omega_{1}$ we have the following property. For almost every $(v, \tau, \xi) \in[0, L] \times[0,1] \times \mathbb{R}$

$$
\left\{\begin{array}{l}
\bar{f}_{\Delta t_{n}}(t, x, v, \tau, \xi)=\bar{f}_{\Delta t_{n}}(t, x, v, 0, \xi-\tau v) \\
\bar{f}_{\Delta t_{n}}(t, x, v, 0, \xi)=\mathcal{M}\left(\bar{f}_{\Delta t_{n}}\left(t-\Delta t_{n}, x, v, 0, \xi-v\right)\right) .
\end{array}\right.
$$


Proof. According to Definition 4.1 and equation (4),

$$
\begin{aligned}
\bar{f}_{\Delta t_{n}}(t, x, v, \tau, \xi) & =f_{\Delta t_{n}}\left(t_{\Delta t_{n}}+\tau \Delta t_{n}, x+\xi \Delta t_{n}, v\right) \\
& =f_{\Delta t_{n}}\left(t_{\Delta t_{n}}, x+\xi \Delta t_{n}-\tau \Delta t_{n} v, v\right) \\
& =f_{\Delta t_{n}}\left(t_{\Delta t_{n}}, x+(\xi-\tau v) \Delta t_{n}, v\right) \\
& =\bar{f}_{\Delta t_{n}}(t, x, v, 0, \xi-\tau v)
\end{aligned}
$$

Moreover,

$$
\begin{aligned}
\bar{f}_{\Delta t_{n}}(t, x, v, 0, \xi) & =f_{\Delta t_{n}}\left(t_{\Delta t_{n}}, x+\xi \Delta t_{n}, v\right) \\
& =f_{\Delta t_{n}}\left(\left(t-\Delta t_{n}\right)_{\Delta t_{n}}+\Delta t_{n}, x+\xi \Delta t_{n}, v\right) \\
& =\mathcal{M}\left(f_{\Delta t_{n}}\left(\left(t-\Delta t_{n}\right)_{\Delta t_{n}}, x+\xi \Delta t_{n}-\Delta t_{n} v, v\right)\right) \\
& =\mathcal{M}\left(f_{\Delta t_{n}}\left(t_{\Delta t_{n}}-\Delta t_{n}, x+(\xi-v) \Delta t_{n}, v\right)\right) \\
& =\mathcal{M}\left(\bar{f}_{\Delta t_{n}}\left(t-\Delta t_{n}, x, v, 0, \xi-v\right)\right),
\end{aligned}
$$

which ends the proof of the proposition.

Now we deduce a property of $\bar{f}_{\Delta t_{n}}$ from the property of $f_{\Delta t_{n}}$ of Proposition 3.2 .

Proposition 5.2. Let $\mathcal{M} \bar{f}_{\Delta t_{n}}$ be the Maxwellian function of $\bar{f}_{\Delta t_{n}}$ with respect to $v$. There exists a subsequence $\Delta t_{n_{p}}$ and a set $\Omega_{2} \subset \Omega_{1}$ (defined in Proposition 5.1) such that $\mathcal{L}\left(\mathbb{R}^{+} \times \mathbb{R} \backslash \Omega_{2}\right)=0$ and for all $(t, x) \in \Omega_{2}$, for all $X>0$, we have

$$
\lim _{p \rightarrow+\infty} \int_{-X}^{X} \int_{0}^{1} \int_{0}^{L}\left|\mathcal{M} \bar{f}_{\Delta t_{n_{p}}}(t, x, v, \tau, \xi)-\mathcal{M} f(t, x, v)\right| d v d \tau d \xi=0 .
$$

Proof. According to Proposition 3.2, for every $R>0$ and $T>0$

$$
\lim _{n \rightarrow \infty} \int_{0}^{T} \int_{-R}^{R} \int_{0}^{L}\left|\mathcal{M} f_{\Delta t_{n}}(t, x, v)-\mathcal{M} f(t, x, v)\right| d v d x d t=0 .
$$

So, according to Proposition 4.1, for all $X>0$,

$$
\lim _{n \rightarrow \infty} \int_{0}^{T} \int_{-R}^{R} \int_{0}^{L} \int_{-X}^{X} \int_{0}^{1}\left|\overline{\mathcal{M}}_{\Delta t_{n}}(t, x, v, \tau, \xi)-\mathcal{M} f(t, x, v)\right| d \tau d \xi d v d x d t=0
$$

But according to Definition 2.1 and Definition 4.1, $\overline{\mathcal{M f}}_{\Delta t_{n}}=\mathcal{M} \bar{f}_{\Delta t_{n}}$. So

$$
\lim _{n \rightarrow \infty} \int_{0}^{T} \int_{-R}^{R}\left\{\int_{0}^{L} \int_{-X}^{X} \int_{0}^{1}\left|\mathcal{M} \bar{f}_{\Delta t_{n}}(t, x, v, \tau, \xi)-\mathcal{M} f(t, x, v)\right| d \tau d \xi d v\right\} d x d t=0
$$


Therefore there exists a subsequence $\Delta t_{n_{p}}$ and a subset $\Omega^{\prime}$ of $\mathbb{R}^{+} \times \mathbb{R}$ such that $\mathcal{L}\left(\mathbb{R}^{+} \times \mathbb{R} \backslash \Omega^{\prime}\right)=0$ and for every $(t, x) \in \Omega^{\prime}$

$$
\lim _{p \rightarrow+\infty} \int_{-X}^{X} \int_{0}^{1} \int_{0}^{L}\left|\mathcal{M} \bar{f}_{\Delta t_{n_{p}}}(t, x, v, \tau, \xi)-\mathcal{M} f(t, x, v)\right| d v d \tau d \xi=0 .
$$

Finally, since $\Omega_{1}$ defined in Proposition 5.1 is such that $\mathcal{L}\left(\mathbb{R}^{+} \times \mathbb{R} \backslash \Omega_{1}\right)=0$, we can take $\Omega_{2}=\Omega_{1} \cap \Omega^{\prime}$.

\section{Proposition 5.3.}

For every $(t, x) \in \Omega_{1}$ there exist a Maxwellian function $g(t, x, \cdot, \cdot) \in L^{\infty}(\mathbb{R} \times$ $[0, L])$ and a subsequence of the sequence $\Delta t_{n_{p}}$, denoted $\Delta t_{n}(t, x)$, which depends on $(t, x)$, such that for every $X>0$

$$
\lim _{n \rightarrow+\infty} \int_{-X}^{X} \int_{0}^{1} \int_{0}^{L}\left|\bar{f}_{\Delta t_{n}(t, x)}(t, x, v, \tau, \xi)-g(t, x, \xi-\tau v, v)\right| d v d \tau d \xi=0 .
$$

Proof. Let us fix a $(t, x) \in \Omega_{1}$. For almost every $(v, \xi) \in[0, L] \times \mathbb{R}$ and for $0 \leq k \leq K$, we denote

$$
m_{k}^{n}(t, x, \xi)=\int_{0}^{L} v^{k} \bar{f}_{\Delta t_{n}}(t, x, v, 0, \xi) d v .
$$

So we have, according to (17) and Definition 2.1,

$$
\begin{aligned}
m_{k}^{n}(t, x, \xi) & =\int_{0}^{L} v^{k} \mathcal{M}\left(\bar{f}_{\Delta t_{n}}\left(t-\Delta t_{n}, x, v, 0, \xi-v\right)\right) d v \\
& =\int_{0}^{L} v^{k} \bar{f}_{\Delta t_{n}}\left(t-\Delta t_{n}, x, v, 0, \xi-v\right) d v .
\end{aligned}
$$

In the computation which follows, we use a simplified notation

$$
\bar{f}_{\Delta t_{n}}(v, \xi)=\bar{f}_{\Delta t_{n}}\left(t-\Delta t_{n}, x, v, 0, \xi\right) .
$$

Thus for every $R>0$ and $0<h<1$,

$$
\begin{aligned}
\int_{-R}^{R} \mid & m_{k}^{n}(t, x, \xi+h)-m_{k}^{n}(t, x, \xi) \mid d \xi \\
& =\int_{-R}^{R}\left|\int_{-\infty}^{\infty} v^{k}\left(\bar{f}_{\Delta t_{n}}(v, \xi+h-v)-\bar{f}_{\Delta t_{n}}(v, \xi-v)\right) d v\right| d \xi \\
& =\int_{-R}^{R}\left|\int_{-\infty}^{\infty}\left((v+h)^{k} \bar{f}_{\Delta t_{n}}(v+h, \xi-v)-v^{k} \bar{f}_{\Delta t_{n}}(v, \xi-v)\right) d v\right| d \xi \\
& \leq C_{R} h+L^{k} \int_{-R}^{R} \int_{-\infty}^{\infty}\left|\bar{f}_{\Delta t_{n}}(v+h, \xi)-\bar{f}_{\Delta t_{n}}(v, \xi)\right| d v d \xi \\
& \leq \tilde{C}_{R} h .
\end{aligned}
$$


In the last step we use that $\bar{f}_{\Delta t_{n}}\left(t-\Delta t_{n}, x, v, 0, \xi\right)$ is Maxwellian with respect to $v$ according to (17) and therefore has a total variation in $v$ uniformly bounded by K. So, using Helly's theorem [6] and a diagonal extraction, there exists a subsequence $\Delta t_{n_{p}}(t, x)$ such that for all $k \in \mathbb{N}$ with $0 \leq k \leq K-1, m_{k}^{n_{p}}(t, x, \cdot)$ converges strongly in $L_{\text {loc }}^{1}(\mathbb{R})$. Thus, since $\bar{f}_{\Delta t_{n}(t, x)}(t, x, \cdot, 0, \cdot)$ is Maxwellian with respect to $v$, according to Proposition 3.1 with $\mathcal{A}=\mathbb{R}$, there exists a Maxwellian function $g(t, x, \cdot, \cdot)$ such that for all $R>0$

$$
\lim _{n \rightarrow+\infty} \int_{-R}^{R} \int_{0}^{L}\left|\bar{f}_{\Delta t_{n}(t, x)}(t, x, v, 0, \xi)-g(t, x, \xi, v)\right| d v d \xi=0 .
$$

So according to the first line of $(17)$, for all $\tau \in[0,1]$

$$
\lim _{n \rightarrow+\infty} \int_{-R}^{R} \int_{0}^{L}\left|\bar{f}_{\Delta t_{n}(t, x)}(t, x, v, \tau, \xi)-g(t, x, \xi-\tau v, v)\right| d v d \xi=0 .
$$

And hence, using the Lebesgue's dominated convergence theorem, we find the result of the proposition.

Proposition 5.4. For all $T>0, R>0$, and $X>0$ we have $\lim _{n \rightarrow+\infty} \int_{-X}^{X} \int_{0}^{1} \int_{0}^{L} \int_{-R}^{R} \int_{0}^{T}\left|\bar{f}_{\Delta t_{n}}(t, x, v, \tau, \xi)-\mathcal{M} f(t, x, v)\right| d t d x d v d \tau d \xi=0$.

Proof. Since $\Omega_{2} \subset \Omega_{1}$, according to Proposition 5.3 and Corollary 3.1, for every $(t, x) \in \Omega_{2}$ and every $X>0$

$$
\lim _{n \rightarrow+\infty} \int_{-X}^{X} \int_{0}^{1} \int_{0}^{L}\left|\mathcal{M} \bar{f}_{\Delta t_{n}(t, x)}(t, x, v, \tau, \xi)-\mathcal{M}(g(t, x, \xi-\tau v, v) t)\right| d v d \tau d \xi=0 .
$$

But, since $\Delta t_{n}(t, x)$ is a subsequence of $\Delta t_{n_{p}}$, according to Proposition 5.2,

$$
\mathcal{M} f(t, x, v)=\mathcal{M}(g(t, x, \xi-\tau v, v))
$$

for almost every $(v, \tau, \xi) \in[0, L] \times[0,1] \times \mathbb{R}$. But the function

$$
(\tau, \xi, v) \longrightarrow g(t, x, \xi-\tau v, v)
$$

lies in $C\left([0,1], L_{\text {loc }}^{1}(\mathbb{R} \times[0, L])\right)$, so according to Corollary 3.1 ,

$$
(\tau, \xi, v) \longrightarrow \mathcal{M}(g(t, x, \xi-\tau v, v))
$$

lies in $C\left([0,1], L_{\text {loc }}^{1}(\mathbb{R} \times[0, L])\right)$ too. Hence for $\tau=0$, we have for all $(t, x) \in \Omega_{2}$ and almost every $(v, \xi) \in[0, L] \times \mathbb{R}$

$$
\mathcal{M}(g(t, x, \xi, v))=\mathcal{M} f(t, x, v) .
$$


But according to Proposition 5.3, $g$ is Maxwellian with respect to $v$. So

$$
g(t, x, \xi, v)=\mathcal{M} f(t, x, v)
$$

which means that $g$ does not depend on the variable $\xi$. So in fact, for almost every $(\tau, \xi, v) \in[0,1] \times \mathbb{R} \times[0, L]$,

$$
g(t, x, \xi-\tau v, v)=\mathcal{M} f(t, x, v) .
$$

Then according to Proposition 5.3, for every $(t, x) \in \Omega_{2}$

$$
\lim _{n \rightarrow+\infty} \int_{-X}^{X} \int_{0}^{1} \int_{0}^{L}\left|\bar{f}_{\Delta t_{n}(t, x)}(t, x, v, \tau, \xi)-\mathcal{M} f(t, x, v)\right| d v d \tau d \xi=0 .
$$

Finally, using the uniqueness of the limit, for the entire sequence $\Delta t_{n_{p}}$

$$
\lim _{p \rightarrow+\infty} \int_{-X}^{X} \int_{0}^{1} \int_{0}^{L}\left|\bar{f}_{\Delta t_{n_{p}}}(t, x, v, \tau, \xi)-\mathcal{M} f(t, x, v)\right| d v d \tau d \xi=0 .
$$

Since $\mathcal{L}\left(\mathbb{R}^{+} \times \mathbb{R} \backslash \Omega_{2}\right)=0$, using the Lebesgue's dominated convergence theorem, we find

$$
\lim _{p \rightarrow+\infty} \int_{0}^{T} \int_{-R}^{R} \int_{-X}^{X} \int_{0}^{1} \int_{0}^{L}\left|\bar{f}_{\Delta t_{n_{p}}}(t, x, v, \tau, \xi)-\mathcal{M} f(t, x, v)\right| d v d \tau d \xi d x d t=0 .
$$

Finally, using again the uniqueness of the limit, the line above holds true for the entire sequence $\Delta t_{n}$.

Now we are able to prove the convergence of the scheme.

Proof of Theorem 1.1. According to Proposition 5.4 and using Proposition 4.1, we find that for all $R>0$ and $T>0$

$$
\int_{0}^{T} \int_{-R}^{R} \int_{0}^{L}\left|f_{\Delta t_{n}}(t, x, v)-\mathcal{M} f(t, x, v)\right| d v d x d t \stackrel{n \mapsto+\infty}{\longrightarrow} 0
$$

which means that $f_{\Delta t_{n}}$ converges strongly to $f$ in $L_{\text {loc }}^{1}\left(\mathbb{R}^{+} \times \mathbb{R} \times[0, L]\right)$ and that $f=\mathcal{M} f$. Finally, let us show that

$$
\lim _{t \mapsto 0}\left\|f(t, \cdot, \cdot)-f^{0}(\cdot, \cdot)\right\|_{L^{1}(\mathbb{R} \times[0, L])}=0 .
$$

First, we have $0 \leq f(t, \cdot, \cdot) \leq 1$. So by weak compactness, there exists a sequence $t_{m}$ and a function $g \in L^{\infty}(\mathbb{R} \times[0, L])$ such that $t_{m}$ converges to 0 when $m$ tends to infinity, $0 \leq g(x, v) \leq 1$, and $f\left(t_{m}, \cdot, \cdot\right)$ converges weakly to $g(\cdot, \cdot)$ in 
$L^{\infty}(\mathbb{R} \times[0, L])$. For $k$ integer, we denote

$$
\begin{aligned}
m_{\Delta t_{n}}^{k}(t, x) & =\int_{0}^{L} v^{k} f_{\Delta t_{n}}(t, x, v) d v \\
m_{0}^{k}(x) & =\int_{0}^{L} v^{k} f^{0}(x, v) d v \\
m^{k}(t, x) & =\int_{0}^{L} v^{k} f(t, x, v) d v .
\end{aligned}
$$

For $0 \leq k \leq K-1$, every function $\varphi \in \mathcal{D}(\mathbb{R})$, and $t>0$, if we multiply equation (9) by $v^{k} \varphi(x)$ and if we integrate on $[0, t] \times \mathbb{R} \times[0, L]$, we find

$$
\int_{\mathbb{R}} \varphi(x) m_{\Delta t_{n}}^{k}(t, x) d x-\int_{\mathbb{R}} \varphi(x) m_{0}^{k}(x) d x=\int_{0}^{t} \varphi^{\prime}(x) m_{\Delta t_{n}}^{k+1}(t, x) d x .
$$

So, passing to the limit when $n$ tends to infinity, we obtain

$$
\begin{aligned}
\left|\int_{\mathbb{R}} \varphi(x) m^{k}(t, x) d x-\int_{\mathbb{R}} \varphi(x) m_{0}^{k}(x) d x\right| & =\left|\int_{0}^{t} \varphi^{\prime}(x) m^{k+1}(t, x) d x\right| \\
& \leq t\left\|\varphi^{\prime}\right\|_{L^{\infty}(\mathbb{R})}\left\|m_{0}^{k+1}\right\|_{L^{1}(\mathbb{R})} .
\end{aligned}
$$

Then when $m$ tends to infinity, $m^{k}(t, \cdot)$ converges weakly to $m_{0}^{k}(\cdot)$ in $\mathcal{D}^{\prime}$, so for almost every $x \in \mathbb{R}$

$$
\int_{0}^{L} v^{k} g(x, v) d v=m_{0}^{k}(x)
$$

Since $f^{0}$ is Maxwellian with respect to $v$, according to Proposition 2.1, for almost every $x \in \mathbb{R}$ we have $\int_{0}^{L} v^{K} g(x, v) d v \geq m_{0}^{K}(x)$ with equality if and only if $g(x, \cdot)=f^{0}(x, \cdot)$. Therefore $\int_{\mathbb{R}} \int_{0}^{L} v^{K} g(x, v) d v d x \geq \int_{\mathbb{R}} m_{0}^{K}(x) d x$ with equality if and only if $g=f^{0}$ almost everywhere. But for almost every $t>0$, if we multiply equation (9) by $v^{K}$ and integrate it on $[0, t] \times \mathbb{R} \times[0, L]$, we have

$$
\int_{\mathbb{R}} \int_{0}^{L} v^{K} f_{\Delta t_{n}}(t, x, v) d v d x \leq \int_{\mathbb{R}} m_{0}^{K}(x) d x .
$$

So when $n$ tends to infinity $\int_{\mathbb{R}} \int_{0}^{L} v^{K} f(t, x, v) d v d x \leq \int_{\mathbb{R}} m_{0}^{K}(x) d x$. Now, when $t_{m}$ tends to 0 we find that $\int_{\mathbb{R}} \int_{0}^{L} v^{K} g(x, v) d v d x \leq \int_{\mathbb{R}} m_{0}^{K}(x) d x$, then we have the equality and $g=f^{0}$. Finally, since $f=\mathcal{M} f$, the values of $f$ are 0 or 1 , so

$$
\begin{aligned}
\left\|f\left(t_{m}, \cdot, \cdot\right)\right\|_{L^{2}(\mathbb{R} \times[0, L])}^{2} & =\int_{\mathbb{R}} \int_{0}^{L} f\left(t_{m}, x, v\right) d x d v=\int_{\mathbb{R}} \int_{0}^{L} f^{0}(x, v) d x d v \\
& =\left\|f^{0}\right\|_{L^{2}(\mathbb{R} \times[0, L])}^{2}
\end{aligned}
$$


The last equality holds true because $f^{0}$ is Maxwellian. Therefore

$$
\lim _{m \mapsto \infty}\left\|f\left(t_{m}, \cdot, \cdot\right)-f^{0}(\cdot, \cdot)\right\|_{L^{1}(\mathbb{R} \times[0, L])}=0 .
$$

But, by uniqueness of the limit, this holds true for every sequence $t_{m}$.

6. Appendix. This appendix is devoted to the proof of Proposition 3.2. We use the averaging lemma of [9].

Proposition 6.1. Let $f_{\Delta t} \in L^{\infty}\left([0, T] \times \mathbb{R}_{x} \times \mathbb{R}_{v}^{+}\right)$satisfying equation (9) for some nonnegative measure $\mu_{\Delta t}$ on $[0, T] \times \mathbb{R}_{x} \times \mathbb{R}_{v}^{+}$, bounded uniformly in $\Delta t$. If $f_{\Delta t}$ is bounded uniformly in $\Delta t$, then $\int_{v} f_{\Delta t}(t, x, v) \psi(v) d v$ belongs to a compact set of $L_{\mathrm{loc}}^{p}\left([0, T] \times \mathbb{R}_{x}\right), 1<p<\infty$, for any $\psi \in L^{p^{\prime}}\left(\mathbb{R}_{v}^{+}\right)$with compact support.

Proof of Proposition 3.2. First we can notice that $f_{\Delta t}$ is solution of the equation

$$
\partial_{t} f_{\Delta t}+v \partial_{x} f_{\Delta t}=\sum_{p} \delta_{p \Delta t}(t)\left[\mathcal{M} f_{\Delta t}-f_{\Delta t}\right]
$$

where $\delta_{p \Delta t}(t)$ is the Dirac measure at $t=p \Delta t$. But according to Proposition 2.1 , for all function $\theta \in C^{\infty}([0, L])$ such that $\partial^{K} \theta(v) / \partial v^{K} \geq 0$

$$
\int_{[0, L]} \theta(v)\left[\mathcal{M} f_{\Delta t}(t, x, v)-f_{\Delta t}(t, x, v)\right] d v \leq 0 .
$$

So there exists a non-negative measure $\mu(t, x, v)$ on $\mathbb{R}^{+} \times \mathbb{R} \times[0, L]$ such that

$$
\sum_{p} \delta_{p \Delta t}(t)\left[\mathcal{M} f_{\Delta t}-f_{\Delta t}\right]=(-1)^{K-1} \partial_{v}^{K} \mu .
$$

Now, if we multiply equation (9) by $v^{K}$ and we integrate it with respect to $(t, x, v)$, we obtain

$$
K ! \int_{0}^{\infty} \int_{0}^{L} \int_{\mathbb{R}} d \mu_{\Delta t}(t, x, v) \leq \int_{0}^{L} \int_{\mathbb{R}} v^{K} f^{0}(x, v) d x d v
$$

which leads to (10) since $v$ lies in $[0, L]$. So by weak compactness, we can find a subsequence $\Delta t_{n}$, a bounded nonnegative measure $\mu(t, x, v)$, and a function $f \in L^{\infty}\left(\mathbb{R}^{+} ; L^{1}(\mathbb{R} \times[0, L])\right)$ such that $\Delta t_{n}$ converges to 0 when $n$ tends to infinity, for almost every $(t, x, v) \in \mathbb{R}^{+} \times \mathbb{R} \times[0, L]$ such that $0 \leq f(t, x, v) \leq 1$ and

$$
\begin{array}{ccc}
f_{\Delta t_{n}} & \stackrel{L^{\infty} \text { weak* }}{\text { measure }} & f \\
\mu_{\Delta t_{n}} & \stackrel{\text { m }}{\text { mat }} & \mu .
\end{array}
$$


Passing to the limit in equation (9), we find

$$
\partial_{t} f+v \partial_{x} f=(-1)^{K-1} \partial_{v}^{K} \mu .
$$

Finally, keeping in mind that the functions $f_{\Delta t}$ have their support in $v$ in $[0, L]$, we can use the Proposition 6.1 with $\psi(v)=v^{k}$ in $[0, L]$ and then we obtain that, up to an extraction, for all integers $0 \leq k \leq K-1$ the function $\int_{0}^{L} v^{k} f_{\Delta t_{n}}(\cdot, \cdot, v) d v$ converges strongly in $L_{\text {loc }}^{1}\left(\mathbb{R}^{+} \times \mathbb{R}\right)$ to $\int_{0}^{L} v^{k} f(\cdot, \cdot, v) d v$. So using Proposition 3.1 with $\mathcal{A}=\mathbb{R}^{+} \times \mathbb{R}$, we obtain that $\mathcal{M} f_{\Delta t_{n}}$ converges strongly in $L_{\text {loc }}^{1}\left(\mathbb{R}^{+} \times \mathbb{R} \times[0, L]\right)$ to $\mathcal{M} f$.

\section{REFERENCES}

[1] V. I. Agoshkov, Spaces of functions with differential-difference charateristics and the smoothnes of solutions of the transport equation, Sov. Math. Dokl. 29 (1984) $662-666$.

[2] Y. BRENIER, personal communication.

[3] _ Résolution d'équations d'évolution quasilináires en dimension $N$ d'espace à l'aide d'équations linéaires en dimension $N+1$, J. Diff. Equ. 50 (1983) 375-390.

[4] _ Averaged multivalued solutions for scalar conservation laws, S.I.A.M. J. Numer. Anal. 21 (1984) 1013-1037.

[5] $\&$ L. CORRIAS, A kinetic formulation for multi-branch entropy solution of scalar conservation laws, Ann. Inst. H. Poincaré Anal. Non Linéaire 15(2) (1998), $169-190$.

[6] H. Brezis, Analyse Fonctionelle, Masson (1987).

[7] A. Chorin, Numerical solutions of the Navier-Stokes equations, Math. Comp. 22 (1968) 745-762.

[8] L. Desvillettes 85 S. Mischler, On the convergence of splitting algorithms for Boltzmann and B. G. K. equations, to appear in Math. Meth. and Mod. Appl. Sc., (1994).

[9] R. J. DiPerna, P. L. Lions $\&$ Y. Meyer, $L^{p}$ regularity of velocity averages, Ann. Inst. Henri Poincaré 8 (1991) 271-287.

[10] Y. Giga 8 T. MiYaKaWa, A kinetic construction of global solutions of first order quasilinear equations, Duke Math. J. 50 (1983) 505-515.

[11] F. Golse, B. Perthame \& 8 R. Sentis, Un résultat de compacité pour les équations de trnasport, C. R. Acad. Sci. Paris 301 (1985) 341-344.

[12] A. Harten, P. LAX $\&$ B. VAN LeER, On upstream differencing and godunov-type schemes for hyperbolic conservation laws, S.I.A.M. Rev. 25 (1983) 35-61.

[13] S. Kaniel \& J. FAlCovitz, Transport approach for compressible flow, in Computing methods in applied sciences and engineering (Proc. Fourth Internat. Sympos. Versailles, 1979) (1980) 133-151.

[14] R. J. LeVeque, Second order accuracy of Brenier's time-discrete method for nonlinear systems of conservation laws, S.I.A.M. J. Num. Anal. 25 (1988) 1-7.

[15] P. L. Lions, B. Perthame \& E. Tadmor, A kinetic formulation of multidimensional scalar conservation laws and related equations, J. Amer. Math. Soc. 7 (1994) 169-191.

[16] Kinetic formulation of the isentropic gas dynamics and p-systems, Commun. Math. Phys. 163 (1994) 415-431. 
[17] D. Serre, Systèmes de Lois de Conservation II. Structures Géométriques, Oscillation et Problèmes mixtes. Fondations, Diderot, Paris (1996) iv+308 pp.

[18] A. VASSEUR, Kinetic semi-discretization of scalar conservation laws and convergence by using averaging lemmas, SINUM (to appear).

Département de Mathématiques et d'Informatique École Normale Supérieure

45 rue d'Ulm

75230 Paris Cedex 05, FRANCE

Also:

Laboratoire d'Analyse Numérique

Université Paris 6

E-MAIL: Alexis.Vasseur@ens.fr

Received: January 6th, 1998. 